RESEÑAS

\title{
La fuerza de la no violencia
}

\author{
Marcos Gutiérrez Gonzáles (iD \\ Universidad de Chile
}

g Judith Butler. La fuerza de la no violencia. Santiago: Paidós, 2020.

Judith Butler es filósofa, ocupa la cátedra Maxine Elliot en el Departamento de Literatura Comparada y el Programa de Teoría Crítica en la Universidad de California, Berkeley. Ha escrito muchas obras relacionadas con los estudios de género, políticas sexuales, derechos humanos y políticas antibélicas.

Este libro trata sobre una forma de entender la no violencia más allá del individualismo y en concordancia con un proyecto que busque una igualdad radical. El primer planteamiento de la autora es que es muy difícil hablar de violencia y no violencia sin dar una definición previa de qué es violento. Además, señala, estos debates para definir la violencia son políticos, porque cada grupo de interés - y el Estado también - va a categorizar como violenta una conducta determinada en atención a sus propios intereses. Un claro ejemplo de esto es la violencia policial que se ejerce contra las personas negras y mestizas en Estados Unidos, ya que estas personas terminan siendo catalogadas como «violentas» y presentadas como amenazas «aun si no están armadas, aun cuando caminan o se escapan, cuando intentan reclamar o simplemente cuando están profundamente dormidas» (página 16). Entonces, en este contexto, intentar la definición de la violencia más amplia y que genere consenso se vuelve imposible. Todo esto, al haber intereses políticos de aumentar el poder estatal y de, por lo tanto, usar esta definición como instrumento para ello.

Este es el punto de partida de este trabajo. Butler busca superar esta discusión aceptando la dificultad de definir la violencia, ya que «está sujeta a cuestiones instrumentales que responden a intereses políticos y, a veces, a la propia violencia del Estado» (página 19). La propuesta pasa a ser, entonces, ir más allá del concepto en sí y de un entendimiento instrumentalista de la violencia para poder pensar, de forma libre, cómo pueden ser las relaciones sociales fuera de la forma dictada por un realismo individualista. En este sentido, se busca proponer una crítica a la violencia desde 
una perspectiva relacional, que pone el acento de los vínculos entre las personas y no tanto en el individuo en sí.

El primer capítulo del libro está dedicado a una crítica a la idea individualista de la no violencia. La idea que trabaja la autora consiste en criticar el entendimiento de la violencia y la no violencia como actos individuales, pasando a proponer que la no violencia implica un compromiso constante con la idea de igualdad. Esta crítica al individualismo comienza como una crítica a la filosofía del contrato social, especialmente sobre la idea de una transición de un estado de naturaleza a la sociedad. Apoyándose en críticas ya existentes a esta corriente de pensamiento, argumenta que las sociedades no nacen producto de un acuerdo entre individuos y que antes, incluso de una posible construcción estatal, ya existen relaciones sociales, ocurriendo en su desarrollo un proceso de individuación, pero que, en ningún momento, este proceso implica una separación del resto de las personas y de las relaciones sociales que nos sustentan.

Asimismo, Butler hace una crítica a la idea de permitir excepciones a la violencia, como los casos en que se permite ejercer violencia con miras a lograr la autopreservación, lo que denomina «legítima defensa». Esto se fundamenta en su idea de igualdad y de que todas las personas dependen de sus vínculos sociales para poder vivir (acá piensa en todos los vínculos posibles, incluidos aquellos necesarios para poder comprar comida y poder vestirse). Entonces, permitir el ejercicio de la violencia para autopreservarse o para preservar a aquellos percibidos como «nosotros» implica una separación, que intenta negar la relación que tienen las personas que ejercen la violencia con aquellos contra quienes se las busca ejercer.

En definitiva, en este capítulo se busca proponer una forma de pensar la igualdad y las relaciones sociales que permita abrirse a la idea de una no violencia comprometida con este proyecto de igualdad, en el cual se busca que todas las personas puedan ser «duelables». ${ }^{1}$

En el segundo capítulo de este libro, Butler avanza el argumento sobre la «duelidad» de las personas. Su objetivo consiste en asentar una crítica a la forma en que se estructura la sociedad actualmente, desde la perspectiva de que no todas las vidas son lamentadas de la misma forma al perderse. El foco está en lo estructural: «mi pregunta no es qué hacemos en cuanto sujetos moralmente responsables, sino cómo está construido el mundo, de modo de reproducir y fortalecer las condiciones necesarias para preservar la vida» (página 91).

De este capítulo vale la pena destacar la idea de que una posición contra la violencia no puede ser ingenua, ya que hay que considerar también el potencial destruc-

1. El concepto de duelidad, conocido como grievability, es concebido por Judith Butler como «el derecho de una persona a tener una vida cuya pérdida merece ser lamentada». Para más información, véase Butler (página 236). 
tivo que existe en las relaciones sociales, un tema que también aborda en el capítulo cuarto. Asimismo, la autora profundiza la idea de que vivir en sociedad implica que nadie puede vivir por si solo ni sustentarse de forma eficaz en dicha condición, por lo que la continuidad de la existencia de las personas depende necesariamente de otras personas. La idea de preservar la vida del otro se funda en que se está vinculado en una relación que hace posible que ambas vidas puedan existir, llegando a proponer la idea de que una sociedad podría estructurarse en base a esas ideas para intentar salvaguardar vidas a través de sus estructuras.

El tercer capítulo busca incorporar una crítica política a su crítica a la violencia. En este momento, la autora se toma de las críticas raciales al orden liberal y de elementos de la biopolítica para ahondar en la idea de que las visiones individualistas sobre la violencia y no violencia están impregnadas de nociones demográficas. Estas nociones implican que, para hablar de violencia legítima y no legítima, el mundo se divide en razas o naciones, entre otras, permitiendo, así, diferenciar a las personas a las que se les ejerce violencia, pudiendo, de esta forma, justificar esta. Esto tiene como correlato que terminan existiendo vidas cuya pérdida no resulta tan valiosa y que, entonces, no serán lamentadas, perpetuando las desigualdades estructurales presentes en la sociedad.

En este capítulo también hay una reflexión sobre la forma en que el Estado y el derecho ejercen violencia y la justifican. Esto tiene lugar a partir de lo escrito sobre la violencia por Walter Benjamin. La idea fundamental en esta parte es que, para hablar de violencia, hay que analizar primero quién es el que está hablando de violencia, en qué contexto y con qué propósitos, de lo contrario no tiene sentido definir la violencia para luego hablar de cuándo es justificable o no. Esto se ve con mayor claridad en aquellos casos en que se denominan como violentas las formas de resistencia no violentas contra la violencia legal, como cuando se tilda de violenta una protesta que consiste en la ocupación de un edificio o una huelga de hambre. En la medida en que esas protestas implican un cuestionamiento a la legitimidad de políticas o gobiernos, son tratadas como violentas por estos últimos por buscar una reestructuración.

Entonces, este capítulo finaliza argumentando que la práctica de la no violencia debe estar basada en un compromiso con la igualdad, porque la perpetuación de la violencia implica una perpetuación de la desigualdad. Además, Butler sostiene acá que, necesariamente, se debe partir de una crítica de la violencia, y que se deben criticar aquellas situaciones en las que se nombra como violenta una acción que cuestiona la legitimidad de un gobierno o de un determinado orden legal, o simplemente una acción de querer cruzar determinadas fronteras. El punto es que esas situaciones invierten la relación de la violencia e invisibilizan a quienes realmente la sufren, a quienes se les ve como la verdadera amenaza. Uno de los ejemplos de esto gira en torno a las personas migrantes que cruzan fronteras y son tildadas como una amenaza para el orden de un determinado país, como una amenaza para la seguridad nacional y 
para las costumbres de ese país, ocultando que son estas personas las que realmente sufrirán la violencia estatal.

En el cuarto capítulo, la reflexión se centra en el plano de lo psicológico. Durante todo el libro, Butler toma referencias del trabajo de Sigmund Freud sobre la pulsión de la muerte y la relación entre el amor y el odio en las relaciones sociales, pero aquí lo analiza con mayor profundidad. La idea central es explorar la relación entre el amor y el odio, entre la constructividad y la destructividad que existe en las personas y en las relaciones sociales. Por un lado, vivimos en un mundo que ejerce violencia y que tiene tendencias destructivas. Por otro lado, señala que Freud teorizó en torno a que las personas tienen una pulsión de muerte y una pulsión de vida. Entonces, en este capítulo se busca trazar una forma de poder construir una ética de la no violencia y una crítica a la violencia que esté comprometida con la idea de igualdad radical a partir de los conceptos desarrollados por Freud.

A partir de la explicación de un intercambio de correspondencia sobre la guerra entre Freud y Albert Einstein en la antesala del régimen nazi, la autora recurre a la idea planteada por el primero sobre la manía. Así, la idea apunta a una especie de freno que hay en la interacción entre el yo y el superyó. El yo busca expresarse violentamente y el superyó controla esta violencia, pero ese control implica una violencia contra uno mismo que puede llevar al extremo de la autodestrucción: el suicidio. Para evitar esta autodestrucción, la manía viene a controlar el pensamiento racional a través de la posibilidad de imaginar otras realidades en que la autopreservación es positiva y, por tanto, se evita el suicidio. En este sentido, la idea de pensar un compromiso con la igualdad radical implica poder pensar más allá de la realidad actual para poder imaginar ese otro mundo y así poder cuestionar la legitimidad del sistema actual. No hay que tomar a la manía como algo bueno en sí, sino como la idea de poder pensar más allá de los que parecen ser los límites de lo posible. Es así como propone que se puede superar el estado de cosas actual, en el que el pensamiento individualista es hegemónico y que se puede comenzar cuestionando este orden de cosas.

En la posdata, cierra con una reflexión sobre la vulnerabilidad y las formas de resistencia a la violencia. Sobre la vulnerabilidad, continúa una crítica que ha sostenido antes sobre la forma en que se ha entendido esta tradicionalmente, como una noción paternalista y que puede invisibilizar los problemas estructurales que la provocan. En este caso, sin embargo, destaca el rol que ha jugado la idea de la vulnerabilidad como herramienta para luchar por los derechos humanos de aquellas personas marginalizadas, solo que cuestiona que ese concepto pueda lograr el cambio que propone en este libro. Acá señala que es importante considerar que la vulnerabilidad es, más bien, un rasgo de las relaciones sociales, ya que esta «solo tiene sentido a la luz de un conjunto de relaciones sociales concretas, que incluyen prácticas de resistencia» (página 221). Entonces, es importante ver cómo la vulnerabilidad también abre un espacio para poder resistir frente a la violencia. Cierra el libro con esta reflexión al 
señalar que una forma no violenta de resistencia implica poner en cuestión el orden social y cuestionar la legitimidad de aquellas prácticas que evitan preservar la vida. En definitiva, señala que la no violencia podrá convertirse en el «deseo por el deseo del otro de vivir» (página 234), es decir, que todas las vidas sean duelables y dignas de ser preservadas.

Sin duda, este libro aporta a la discusión sobre la violencia, muy contingente en la actualidad y visibiliza las formas en las cuales vale la pena reflexionar en torno a ella. Esta crítica a la violencia viene aparejada de un compromiso con la igualdad y la no violencia y, además, permite intentar ir más allá de la discusión sobre qué violencia es legítima para poder construir un proyecto en el cual todas las personas puedan vivir vidas consideradas como dignas de preservar y lamentar. Esto, teniendo siempre en consideración la real posibilidad de conflicto, pero considerando las formas de solucionarlo que no impliquen un desconocimiento de la duelidad de otros.

\section{Sobre el autor}

Marcos Gutiérrez González es egresado de la carrera de Derecho de la Universidad de Chile. Además, fue ayudante del curso de Constitucionalismo y Codificación en los Siglos XIX y XX. Por otra parte, cursó un intercambio estudiantil en la Universidad de Paris I Panthéon-Sorbonne, Francia. Actualmente es ayudante del Centro de Derechos Humanos, de un curso de derecho internacional de los derechos humanos y de un curso de derecho administrativo, todos de la Facultad de Derecho de la Universidad de Chile. Su correo electrónico es marcos.gutierrez@derecho.uchile.cl. (D) https://orcid.org/oooo-0001-5242-2238. 
